13

\title{
Об адсорбции газов на карбиде кремния: простые оценки
}

\author{
() С.Ю. Давыдов ${ }^{1}$, О.В. Посредник ${ }^{2}$ \\ ${ }^{1}$ Физико-технический институт им. А.Ф. Иофффе РАН, \\ Санкт-Петербург, Россия \\ ${ }^{2}$ Санк-Петербургский государственный электротехнический университет (ЛЭТИ), \\ Санкт-Петербург, Россия \\ E-mail: Sergei_Davydov@mail.ru
}

Поступила в Редакцию 19 марта 2019 г.

В окончательной редакции 19 марта 2019 г.

Принята к публикации 2 апреля 2019 г.

\begin{abstract}
В рамках двух физически различных подходов (твердотельном и квантово-химическом) рассмотрена адсорбция атомарного азота и молекул азота и аммиака на карбиде кремния. В твердотельной подходе с использованием модели Халдейна-Андерсона для плотности состояний $4 H$ и $6 H$ политипов $\mathrm{SiC}$ показано, что энергии связи атомов $\mathrm{N}$ и молекулы $\mathrm{N}_{2}$ с подложкой равны 6 и $3 \mathrm{eV}$ соответственно. В квантово-химическом подходе в модели двухатомной поверхностной молекулы для энергии связи атомарного азота получены величины, равные $6 \mathrm{eV}$ для адсорбции на С-грани и $4 \mathrm{eV}$ для адсорбции на $\mathrm{Si}$-грани. Установлено, что во всех рассмотренных случаях переходом заряда между адсорбатом и подложкой можно пренебречь. Высказано предположение, что, как и в случае адсорбции аммиака на $\operatorname{Si}(100)$, для карбида кремния имеет место диссоциация молекулы с последующей пассивацией оборванных $s p^{3}$-орбиталей карбида кремния атомами водорода.
\end{abstract}

Ключевые слова: модель Халдейна-Андерсона, модель поверхностной молекулы, переход заряда, энергия адсорбции.

DOI: 10.21883/FTT.2019.08.47985.429

\section{1. Введение}

Карбид кремния привлекает внимание исследователей, прежде всего, как широкозонный материал с повышенной стойкостью к температурным, механическим и радиационным воздействиям, что позволяет использовать приборы на его основе в экстремальных условиях [1-3]. В последнее десятилетие карбид кремния обрел новую область применения в качестве исходного объекта для получения углеродных наноструктур [4,5]. Недавно предложен также оригинальный способ получения $\mathrm{SiC}$ из кремния (метод сборки) [6]. В свете сказанного, представляет интерес вопрос об адсорбционной способности карбида кремния. В настоящей работе мы приведем теоретические оценки перехода заряда и энергии связи некоторых атомов и молекул с поверхностью карбида кремния. Отметим, что помимо популярных и широко применяемых в настоящее время расчетов из первых принципов (в основном, в рамках различных вариантов функционала плотности), к задаче об адсорбции существуют и модельные походы. Здесь наиболее последовательным является приближение, основанное на модели Халдейна-Андерсона [7-9]. Идеологически близкий, но упрощенный подход состоит в использовании модифицированной модели Андерсона $[10,11]$. И, наконец, рассматривая адсорбцию в режиме поверхностной молекулы [7], можно получить простые оценки, воспользовавшись методом связывающих орбиталей Харрисона [12]. В настоящей работе используются первый и третий подходы. В качестве адсорбатов рассмотрены $\mathrm{N}_{2}$ и $\mathrm{NH}_{3}$ и атомы N. Отметим, что проблема взаимодействия газов $\mathrm{N}_{2}$ и $\mathrm{NH}_{3}$ с поверхностью $\mathrm{SiC}$ возникла в связи с изучением интерфейса $\mathrm{SiO}_{2} / \mathrm{SiC}$ [13-17]. При этом имеет место диссоциация молекул. Найти какие-либо работы непосредственно по адсорбции $\mathrm{N}_{2}, \mathrm{~N}$ и $\mathrm{NH}_{3}$ на $\mathrm{SiC}$ не удалось. Поэтому ниже мы будем основываться на косвенных данных и некоторых предположениях, так как экстраполировать на случай поверхности результаты работ [13-17], полученные для объема, не корректно.

\section{2. Модель Халдейна-Андерсона}

\section{1. Общие соотношения}

Из самых общих соображений [7-9] функция Грина $G_{a}(\omega)$ для адсорбированной частицы (адчастицы) может быть записана в виде

$$
G_{a}^{-1}(\omega)=\omega-\varepsilon_{a}-\Lambda_{a}(\omega)+i \Gamma_{a}(\omega) .
$$

Здесь $\omega-$ энергетическая переменная, $\varepsilon_{a}-$ энергия одноэлектронного уровня адчастицы; $\Gamma_{a}(\omega)=$ $=\pi V_{a}^{2} \rho_{s u b}(\omega)-$ функция уширения квазиуровня адчастицы, где $\rho_{s u b}(\omega)$ - плотность состояний $(П С)$ субстрата, $V_{a}$ - матричный элемент взаимодействия адчастица-подложка; $\Lambda_{a}(\omega)=\frac{1}{\pi} P \int_{-\infty}^{\infty} \frac{\Gamma_{a}\left(\omega^{\prime}\right) d \omega^{\prime}}{\omega-\omega^{\prime}}-$ функция сдвига квазиуровня, где $P-$ символ главного значения. Плотность состояний (ПС) на адчастице. Плотность 
состояний на адчастице $\rho_{a}(\omega)$, отвечающая функции Грина (1), имеет вид

$$
\rho_{a}(\omega)=\frac{1}{\pi} \frac{\Gamma_{a}(\omega)}{\left[\omega-\varepsilon_{a}-\Lambda_{a}(\omega)\right]^{2}+\Gamma_{a}^{2}(\omega)},
$$

а число заполнения $n_{a}$ уровня $\varepsilon_{a}$ адчастицы при нулевой температуре равно

$$
n_{a}=\int_{-\infty}^{E_{F}} \rho_{a}(\omega) d \omega,
$$

где $E_{F}$ - уровень Ферми подложки.

Энергия адсорбции $-E_{a d s}=E_{a d s}^{m e t}+E_{s u b}^{i o n}$, где первое слагаемое есть металлическая составляющая энергии адсорбции, вторая - ионная составляющая. Можно показать $[7,8]$, что

$$
E_{a d s}^{m e t}=\int_{-\infty}^{E_{F}}\left(\omega-E_{F}\right) \Delta \rho_{s y s}(\omega) d \omega
$$

где $\Delta \rho_{s y s}=\rho_{s y s}-\rho_{s y s}^{0}$ и $\rho_{s y s}^{0}$ и $\rho_{s y s}$ есть ПС системы до и после адсорбции. Ионная составляющая может быть оценена выражением

$$
E_{a b s}^{i o n}=-\frac{\left(Z_{a} e\right)^{2}}{4 d}
$$

где $Z_{a}$ - заряд адчастицы, равный $1-n_{a}$, если изначально орбиталь была заполнена, и $\left(-n_{a}\right)$, если орбиталь пуста, $d$ - длина адсорбционной связи.

Отметим, что здесь и далее мы рассматриваем одноэлектронную (или однодырочную) частицу, которая в силу внутриатомного кулоновского отталкивания может содержать на внешней орбитали лишь один электрон (одну дырку) [7-9].

Для описания полупроводниковой подложки весьма удобна простая модель Халдейна-Андерсона [7$9]$, в которой $\rho_{s u b}(\omega)=\rho_{s}$ для $\left|\omega-E_{0}\right| \geq E_{g} / 2$ и $\rho_{s u b}(\omega)=0$ для $\left|\omega-E_{0}\right|<E_{g} / 2$, где $E_{0}=\chi+E_{g} / 2-$ центр запрещенной зоны относительно вакуума, $\chi-$ электронное сродство политипа карбида кремния. Тогда $\quad \Gamma_{s}=\pi V_{a}^{2} \rho_{s}=\mathrm{const}, \quad \Lambda(\omega)=(\Gamma / \pi) \ln \mid\left(\omega-E_{0}\right.$ $\left.-E_{g} / 2\right) /\left(\omega-E_{0}+E_{g} / 2\right) \mid$. При этом, в отсутствии вырождения для полупроводниковой подложки, число заполнения адчастицы $n_{a}=n_{v}+n_{l}$ [7-9], где вклад валентной зоны, согласно (2), есть

$$
n_{v}=\int_{-\infty}^{-E_{g} / 2} \rho_{a}(\omega) d \omega
$$

а вклад локального состояния

$$
n_{l}=\left(1+\frac{1}{\pi} \frac{\Gamma_{s} E_{g}}{\left(E_{g} / 2\right)^{2}-\omega_{l}^{2}}\right)^{-1} \cdot \Theta\left(E_{F}-\omega_{l}\right),
$$

где $\omega_{l}-$ энергия лежащего в запрещенной зоне локального состояния, являющаяся корнем уравнения $\omega-\varepsilon_{a}-\Lambda_{a}(\omega)=0$ в интервале $|\omega|<E_{g} / 2, \Theta(\ldots)-$ функция Хэвисайда.

\section{2. Адсорбция молекулы $\mathbf{N}_{2}$}

Рассмотрим адсорбцию молекулы $\mathrm{N}_{2}$ на $\mathrm{SiC}$. Coгласно [18], для политипов $4 H-$ и $6 H-\mathrm{SiC}$ электронное сродство $\chi$ и ширина запрещенной зоны $E_{g}$ равны соответственно $3.17,3.23 \mathrm{eV}$ и $3.45,3.00 \mathrm{eV}$. В дальнейшем за нуль энергии примем положение центра запрещенной зоны относительно вакуума: $E_{0}=\chi+E_{g} / 2$. Энергия ионизации молекулы $\mathrm{N}_{2}$ равна $I=15.58 \mathrm{eV}$ [19]. Энергия квазиуровня $\varepsilon_{a}=-I+e^{2} / 4 d+E_{0}$, где второе слагаемое описывает кулоновский сдвиг квазиуровня адчастицы $[7,8]$. Полагая $d=1 \AA$ (порядка межатомного расстояния в молекуле $\mathrm{N}_{2}[20]$ ), получим $\varepsilon_{a}=-7.20 \mathrm{eV}$ $(4 \mathrm{H}-\mathrm{SiC})$ и $\varepsilon_{a}=-7.03 \mathrm{eV}(6 \mathrm{H}-\mathrm{SiC})$. Ясно, что столь глубоко залегающие уровни содержат 1 электрон, так что заряд у адмолекулы практически отсутствует, и ионная составляющая (4) энергии адсорбции $E_{a d s}^{i o n} \sim 0$.

Действительно, оценим значения вкладов валентной зоны $n_{v}(5)$ в числа заполнения по формуле $n_{a}$ (см. [9]):

$$
n_{v}=\frac{1}{\pi} \operatorname{arcctg} \frac{\varepsilon_{a}+R}{\Gamma_{s}}, \quad R=\frac{E_{g}}{4} \sqrt{1+\frac{8 \Gamma_{s}}{\pi E_{g}}} .
$$

При $\Gamma_{s}=0.5 \mathrm{eV}$ из (7) получим $n_{v} \sim 1$ как для 4H-SiC, так и для $6 \mathrm{H}-\mathrm{SiC}$. С другой стороны, $n_{l} \sim 0$ (см. (6) и рис. 1 в [9]), так что $n_{a} \sim 1$ и $Z_{a} \sim 0$.

Для оценки металлической (или ковалентной) составляющей энергии адсорбции $E_{a d s}^{m e t}(3)$ воспользуемся соотношением неопределенности $\Delta x \cdot \Delta p \sim \hbar$. Полагая, что в изолированной молекуле $\Delta x \sim d$, а в адсорбированном состоянии $\Delta x \sim 2 d$, получим выигрыш в кинетической энергии $\Delta E_{k i n} \sim 3 \hbar^{2} / 8 m d^{2} \approx 3 \mathrm{eV} \sim\left|E_{a d s}^{m e t}\right|$, где $m-$ масса свободного электрона.

Отметим, что при использовании модели ХалдейнаАндерсона различия в адсорбции на $\mathrm{Si}$ - и С-гранях входят только через матричный элемент $V_{a}$, и, следовательно, $\Gamma_{s}$. Если в рамках твердотельного подхода считать, что длины адсорбционной связи для $\mathrm{Si}-$ и С-граней одинаковы, то различия вообще исчезают (см., однако, ниже раздел 2).

Как уже отмечалось, нам, к сожалению, не удалось отыскать в литературе данных по энергии адсорбции молекулы $\mathrm{N}_{2}$ на $\mathrm{SiC}$. По данным [21] молекула $\mathrm{N}_{2}$ не диссоциирует на W(110). Мы в приведенных оценках также предполагали, что на $\mathrm{SiC}$ адсорбируется именно молекула, а не атомы азота.

\section{3. Адсорбция молекулы $\mathrm{NH}_{3}$ и атома $\mathrm{N}$}

В случае адсорбции молекул $\mathrm{NH}_{3}$ на $\mathrm{Si}(100)$ имеет место диссоциация молекулы [21]. Возникающие при этом атомы водорода пассивируют оборванные $s p^{3}$-орбитали кремния, прекращая реакцию. Нет каких-либо оснований полагать, что на Si- и C-гранях карбида кремния дело обстоит иначе. Таким образом, адсорбция аммиака в молекулярном виде на карбиде кремния не происходит.

Рассмотрим теперь адсорбцию атомарного азота на карбиде кремния. Энергия ионизации атома азота 
$I=14.53 \mathrm{eV}$ [19], что мало отличается от энергии ионизации молекулы $\mathrm{N}_{2}$. Вновь полагая $d=1 \AA$ (порядка межатомного расстояния в молекуле $\mathrm{N}_{2}$ [20]), получим $\varepsilon_{a}=-6.15 \mathrm{eV}(4 \mathrm{H}-\mathrm{SiC})$ и $\varepsilon_{a}=-5.98 \mathrm{eV}(6 \mathrm{H}-\mathrm{SiC})$. Это, также как и в случае $\mathrm{N}_{2} / \mathrm{SiC}$, глубокие уровни, так что переходом заряда можно пренебречь. Таким образом, ионная составляющая энергии адсорбции $E_{a d s}^{i o n} \sim 0$. Для выигрыша в кинетической энергии вновь имеем выражение $\Delta E_{k i n} \sim 3 \hbar^{2} / 8 m d^{2}$, где $d$ можно положить равным атомному радиусу азота $r_{\mathrm{N}}=0.71 \AA$. Тогда получаем $\Delta E_{k i n} \sim 6 \mathrm{eV} \sim\left|E_{a d s}^{m e t}\right|$.

\section{3. Метод связывающих орбиталей}

Подход к задаче об адсорбции, рассмотренный в предыдущем разделе, можно назвать твердотельным, эквивалентным задаче о примеси в твердотельной матрице. В этом разделе мы используем квантово-химическое приближение к проблеме, в рамках которого рассматривается кластер, состоящий из адсорбированной частицы и ближайших к ней атомов подложки. В предельном случае такой кластер можно свести к двухатомной молекуле, состоящей из адатома и связанного непосредственно с ним атома подложки (модель поверхностной молекулы) [7].

Начнем с рассмотрения адсорбции атома азота на С-грани SiC. В рамках метода связывающих орбиталей $(\mathrm{MCO})[12]$, получим длину связи $\mathrm{C}-\mathrm{N}$ равной $d_{\mathrm{CN}}=r_{\mathrm{N}}+r_{\mathrm{C}}=1.48 \AA$, где атомный радиус углерода $r_{\mathrm{C}}=0.77 \AA$ [19]. Для $\sigma$-связи $s p^{3}$-орбитали углерода с $p$-орбиталью азота ковалентная энергия связи равна $V_{2}=\left(\hbar^{2} / m d_{\mathrm{CN}}^{2}\right)\left(\eta_{s p \sigma}+\sqrt{3} \eta_{p p \sigma}\right) / 2$ [22], где $\eta_{s p \sigma}=1.42$, $\eta_{p p \sigma}=2.22[12,23]$, откуда получаем $V_{2}(\mathrm{CN})=9.16 \mathrm{eV}$. Энергия гибридизованной $s p^{3}$-орбитали углерода равна $-\varepsilon_{h}(\mathrm{C})=\left(\varepsilon_{s}+3 \varepsilon_{p}\right) / 4=13.15 \mathrm{eV}$, а энергия $p$-орбитали азота есть $s_{p}=-13.84 \mathrm{eV}$ (таблицы атомных термов Манна $[12,24])$. Отсюда полярная энергия связи $\mathrm{C}-\mathrm{N}$, определяемая как $V_{3}(\mathrm{CN})=\left(\varepsilon_{h}(\mathrm{C})-\varepsilon_{p}(\mathrm{~N})\right) / 2$ $=0.35 \mathrm{eV}$. Тогда ковалентность связи $\alpha_{c}=V_{2} / \sqrt{V_{2}^{2}+V_{3}^{2}}$ $\approx 1$. В простейшем виде энергию связи можно представить в виде

$$
E_{b} \approx \frac{2 V_{2}}{\alpha_{c}}\left(1-\frac{2}{3} \alpha_{c}^{2}\right)
$$

(см., например, формулу (3) работы [25], где, однако, мы поменяли знак $\left.E_{b}\right)$, откуда находим $E_{b}(\mathrm{CN}) \approx 2 V_{2} / 3 \approx 6 \mathrm{eV}$. Отметим, что в данной модели мы считаем $E_{b}=-E_{a d s}$, так что полученное нами значение $E_{b}(\mathrm{CN})$ совпадает с результатом раздела 2 . То же относится и к переходу заряда, который, в рамках МСО, оценивается величиной полярности связи $\alpha_{P}=\sqrt{1-\alpha_{c}^{2}} \ll 1$.

Перейдем теперь к адсорбции атомов азота на Si-грани SiC. Так как $r_{\mathrm{Si}}=1.18 \AA$, имеем $d_{\mathrm{SiN}}=r_{\mathrm{N}}+r_{\mathrm{Si}}$ $=1.89 \AA \quad$ и $\quad V_{2}(\mathrm{SiN})=5.62 \mathrm{eV}$. Так как $-\varepsilon_{h}(\mathrm{Si})=$ $=\left(\varepsilon_{s}+3 \varepsilon_{p}\right) / 4=9.39 \mathrm{eV}, \quad$ получаем $\quad V_{3}(\mathrm{SiN})=\left(\varepsilon_{h}(\mathrm{Si})\right.$ $\left.-\varepsilon_{p}(\mathrm{~N})\right) / 2=2.23 \mathrm{eV}, \alpha_{c} \approx 0.97$ и $E_{b}(\mathrm{SiN}) \approx 4 \mathrm{eV}$. Таким образом, энергия связи атома азота с С-гранью в полтора раза больше, чем с Si-гранью. В обоих случаях связь практически гомополярная.

\section{4. Заключительные замечания}

Итак, в настоящей работе для оценок перехода заряда между адчастицей и $\mathrm{SiC}$-подложкой и значений энергии адсорбции использованы две принципиально различные модели: 1) твердотельная модель, в которой явным образом учитывается наличие запрещенной зоны подложки, но игнорируются природа адсорбирующей грани (C или $\mathrm{Si})$ и геометрия адсорбционного комплекса, и 2) модель поверхностной молекулы, где зонная структура подложки вообще не учитывается, но принимается во внимание различие длин адсорбционной связи на C- и Si-гранях. Сопоставление полученных в различных моделях результатов показывает хорошее соответствие. Помимо близких значений энергий адсорбции, следует указать на совпадение оценок для практически нулевого перехода заряда между адсорбатом и адсорбентом. Интересно отметить, что при адсорбции газов на $d$-металлах переход заряда также крайне мал [26]. Стоит подчеркнуть также, что оба использованных здесь простых приближения достаточно легко могут быть модифицированы для более строгого учета особенностей адсорбционной системы (см., например, [27,28]).

\section{Благодарности}

Авторы признательны С.А. Кукушкину за предложение темы и полезные обсуждения.

\section{Конфликт интересов}

Авторы заявляют, что у них отсутствует конфликт интересов.

\section{Список литературы}

[1] Silicon Carbide: recent major advances / Ed. W.J. Choyke, H. Matsunami, G. Pensl. Berlin-Heidelberg, Springer (2004). http://www.springer.de.

[2] Advances in Silicon Carbide. Processing and Applications / Ed. S.E. Saddow, A. Agarwal. Boston-London, Artech House (2004). www.artechhouse.com.

[3] A.A. Lebedev. Semicond. Sci. Technol. 21, R17 (2006).

[4] Y.H. Woo, T. Yu, Z.X. Chen. Appl. Phys. Rev. 108, 071301 (2010).

[5] Г.В. Бенеманская, П.А. Дементьев, С.А. Кукушкин, А.В. Осипов, С.Н. Тимошнев. Письма в ЖТФ 45, 5, 17 (2019).

[6] С.А. Кукушкин, А.В. Осипов, Н.А. Феоктистов. ФТТ 56, 1457 (2014).

[7] С.Ю. Давыдов. Теория адсорбции: метод модельных гамильтонианов. СПб.: Изд-во СПбГЭТУ „ЛЭТИ“(2013). 235 c. twirpx.com/file/1596114/. 
[8] С.Ю. Давыдов, А.А. Лебедев, О.В. Посредник. Элементарное введение в теорию наносистем. Изд-во „Лань“, СПб (2014).

[9] С.Ю. Давыдов, С.В. Трошин. ФТТ 49, 1508 (2007).

[10] С.Ю. Давыдов, А.В. Павлык. ФТП 35, 831 (2001).

[11] С.Ю. Давыдов, А.В. Павлык. Письма в ЖТФ 29, 12, 33 (2003).

[12] С.Ю. Давыдов, О.В. Посредник. Метод связывающих орбиталей в теории полупроводников. Учеб. пособие. Изд-во СПбГЭТУ „ЛЭТИ“, СПб (2007). 96 с. (twirpx.com/file/1014608/).

[13] J.P. Xu, P.T. Lai, C.L. Chan, Y.C. Cheng. Appl. Phys. Lett. 76, $372(2000)$.

[14] Y.S. Liu, S. Hashimoto, K. Abe, R. Hayashibe, T. Yamakami, M. Nakao, K. Kamimura. Jap. J. Appl. Phys. 44, 673 (2005).

[15] Y. Iwasaki, H. Yano, T. Hatayama, Y. Uraoka, T. Fuyuki. Appl. Phys. Express 3, 026201 (2010).

[16] F. Liu, C. Carraro, A.P. Pisano, R. Maboudian. J. Micromech. Microeng. 20, 035011 (2010).

[17] E. Pitthan, A.L. Gobbi, H.I. Boudinov, F.C. Stedile. J. Electronic Mater. 44, 2823 (2009).

[18] С.Ю. Давыдов. ФТП 53, 706.(2019).

[19] Физические величины. Справочник / Под ред. Е.С. Григорьева, Е.3. Мейлихова. Энергоатомиздат, М (1991).

[20] Tables of Interatomic Distances and Configuration in Molecules and Ions / Ed. L.E. Sutton. The Chemical Society, London (1958).

[21] M.D. Ramsier, J.T. Yates, Jr. Surf. Sci. Rep. 12, 243 (1991).

[22] С.Ю. Давыдов, С.К. Тихонов. ФТТ 37, 2749 (1995).

[23] W.A. Harrison. Phys. Rev. B 27, 3552.( 1983).

[24] W.A. Harrison. Phys. Rev. B 31, 2121 (1985).

[25] С.Ю. Давыдов, О.В. Посредник. ФТТ 57, 819 (2015).

[26] Л.А. Большов, А.П. Напартович, А.Г. Наумовец, А.Г. Федорус. УФН 122, 125 (1977).

[27] С.Ю. Давыдов. ЖТФ 84, 4, 155 (2014).

[28] С.Ю. Давыдов, А.В. Зубов, А.А. Лебедев. Письма в ЖТФ 45, 9, 40 (2019).

Редактор Т.Н. Василевская 